\title{
ESTILOS GERENCIALES Y SATISFACCIÓN LABORAL
}

\author{
MANAGEMENT STYLES AND JOB SATISFACTION
}

\author{
Valencia Pomareda, Pedro Herbert Jaime* \\ hervalpo@gmail.com
}

[RECEPCIÓN: JULIO DEL 2014/CONFORMIDAD: SETIEMBRE DEL 2014]

\section{RESUMEN}

Desde los experimentos de Howthorne, la administración continuó buscando la mejor manera de superar las relaciones que se tienen con los colaboradores. Es así que siendo el Liderazgo un elemento importante en la gestión estratégica, escogimos entre los muchos estilos de liderazgo el de Rensis Likert, por ser el más técnico y apropiado para logran con mayor efectividad el empowerment, estableciendo el grado de satisfacción que los estilos de Liderazgo de Liker buscan en nuestros colaboradores. Es por ello que buscamos conocer el nivel de satisfacción laboral que los estilos de liderazgo de Likert, logran a través de la teoría de Herzberg.

Palabras claves: Estilos de gestión, satisfacción laboral.

\begin{abstract}
From Howthorne experiments, the administration continued looking for the best way to improve the relationships they have with reviewers, is so important to be Leadership in strategic management element, chose among the many leadership styles for the Rensis Liker be the most appropriate technical and more effectively to achieve empowerment, establishing satisfaction that leadership styles Likert looking at our employees, which is why we seek to know the level of job satisfaction that leadership styles Likert, achieved through Herzberg.
\end{abstract}

Keywords: Management styles, job satisfaction.

\footnotetext{
* Magíster en Administración. Docente asociado e investigador de la Facultad de Ciencias Administrativas de la Universidad Nacional Mayor de San marcos
} 


\section{INTRODUCCIÓN}

Gestionar personas se constituye hoy en día en uno de los aspectos claves de la función gerencial. Si los gerentes modernos cuentan con equipos humanos de alta eficiencia, que posean además adecuados niveles de motivación y compromiso con su institución, existirán mayores posibilidades de delegar funciones a personal competente y motivado para desarrollar un buen trabajo y brindar un servicio de calidad. Esto conduce además a que los gerentes liberen tiempo para diseñar y ejecutar nuevos planes y estrategias de desarrollo institucional.

Uno de los aspectos clave en la gestión de personas en las instituciones modernas es el manejo de un estilo adecuado de liderazgo. Este tema muchas veces ha permanecido ignorado o soslayado debido a que los propios gerentes no conocían su existencia y mucho menos que era un manejo importante para facilitar y optimizar su gestión.

\section{PLANTEAMIENTO DEL PROBLEMA PROBLEMA GENERAL}

¿Cómo se relacionan los estilos gerenciales con la satisfacción laboral en el personal administrativo de la Dirección General de Administración de la UNMSM en el año 2008?

\section{PROBLEMAS ESPECÍFICOS}

¿Qué grado de asociación existe entre los estilos gerenciales autoritario-coercitivo y autoritario-benevolente y la satisfacción laboral en el Personal Administrativo de la Dirección General de Administración de la UNMSM en el año 2008?

¿Qué grado de asociación existe entre los estilos gerenciales consultivo y participativo y la satisfacción laboral en el personal administrativo de la Dirección General de Administración de la UNMSM en el año 2008?

\section{OBJETIVOS DE LA INVESTIGACIÓN}

\section{Objetivo general}

Determinar cómo se relacionan los estilos gerenciales con la satisfacción laboral en el personal administrativo de la Dirección General de Administración de la UNMSM.

\section{Objetivos específicos}

Determinar el grado de asociación existente entre el estilo gerencial autoritario-coercitivo y autoritario-benevolente con los factores motivadores e higiénicos de la satisfacción laboral en el personal administrativo de la Dirección General de Administración Central de la UNMSM.

Determinar el grado de asociación existente entre el estilo gerencial consultivo y participativo con los factores motivadores e higiénicos de la satisfacción laboral en el personal administrativo de la Dirección General de Administración UNMSM.

\section{JUSTIFICACIÓN}

Como un proceso natural, los buenos resultados se deben obtener como una consecuencia de la satisfacción positiva de las personas en el trabajo.

Así como la cultura de un país da forma a los valores de sus habitantes, la cultura de una organización regula el concepto de satisfacción laboral. Esto significa que así como los valores no son totalmente universales sino que se van aplicando y priorizando de acuerdo a la realidad de cada país, la satisfacción laboral se va logrando en correspondencia con la realidad de cada organización y lo que esta ofrece a los trabajadores que la integran.

De esta realidad se deriva también lo que las organizaciones están dispuestas a pagar en correspondencia con la facilidad o dificultad que encuentran para captar a la fuerza laboral que necesitan. El hecho de que esta fuerza laboral haya crecido en calidad y cantidad en los últimos años no ha corrido en paralelo con la generación de nuevos puestos de trabajo, lo que ha originado ciertas distorsiones en el mercado salarial.

Los empleadores, al poder acceder actualmente con más facilidad a una oferta laboral calificada, ya no se encuentran tan dispuestos a conceder los mismos incentivos monetarios que otorgaban antes para retener y satisfacer las necesidades de los trabajadores.

Es por este motivo que nos hemos permitido escoger como variable independiente la teoría de los factores de Herzberg (1966), donde el autor considera a los factores motivacionales o intrínsecos como poderosos incentivadores de satisfacción positiva con la organización, con el trabajo y consigo mismo, contraponiéndolos a 
los factores extrínsecos o higiénicos que causan mayores costos y no originan necesariamente satisfacción laboral.

El mismo Frederick Herzberg (1966) manifiesta que las necesidades de superación y la de realizar un trabajo que se valora constituyen la mayor fuente de satisfacción laboral, reportando también que las relaciones interpersonales en el trabajo y la oportunidad que este brinda para progresar, los que componen los factores motivacionales de la teoría de Herzberg (1966) fueron consideradas más importantes que el dinero.

La teoría de Herzberg (1966) formula además que las altas remuneraciones no estimulan por sí solas al trabajador para desempeñarse con eficiencia en el trabajo. Aunque los trabajadores aspiran obtener unos ingresos justos y equilibrados, solo esperan recibir lo que se paga a otros por trabajos análogos y aunque podría quedar insatisfecho si se le remunera insuficientemente, un sueldo más alto no garantiza por sí solo la satisfacción plena en el empleo. En cambio, la estabilidad o continuidad en el empleo parece un estímulo primordial, especialmente cuando esta surge en razón de la buena ejecución de las tareas. Otros aspectos importantes son el tipo de trabajo, las oportunidades de ascenso y la relación con los compañeros. También, las condiciones satisfactorias del trabajo se perciben ligadas probablemente al deseo de ser tratados con humanidad por los superiores.

Ahora bien, si los buenos resultados se deben obtener como consecuencia de la satisfacción positiva de las personas en el trabajo, y como por la recesión por la que atraviesan las empresas no es posible otorgar incentivos económicos suficientes al trabajador (en la administración pública, no es posible por razones presupuestales), entonces por qué no buscar otro tipo de incentivos que estimulen a los colaboradores a cumplir mejor con su trabajo, y estos los encontramos en los factores motivacionales de Herzberg (1966).

La satisfacción laboral debe entenderse como un estado emocional positivo que refleja una respuesta afectiva frente a los aspectos o facetas de trabajo.

Al aplicar los estilos gerenciales de Rensis Likert (1968) y relacionarlos con los factores de satisfacción laboral de Herzberg (1966), obtendremos resultados que nos permitirán precisar recomendaciones para mejorar las variables de resultado (productividad, beneficios, ventas, etc) de los estilos gerenciales de Rensis Likert (1968), pudiéndose complementar este beneficio a otras dependencias de la universidad, otras empresas u organizaciones relacionadas.

\section{HIPÓTESIS}

\section{HIPÓTESIS GENERAL}

Existe relación significativa entre los estilos gerenciales y la satisfacción laboral en el personal administrativo de la Dirección General de Administración de la UNMSM.

\section{METAS ESPECÍFICAS}

Existe un grado de asociación poco significativo entre el estilo gerencial autoritario explotador y autoritario benevolente, con los factores motivadores e higiénicos de la satisfacción laboral en el personal administrativo de la Administración Central de la UNMSM.

Existe un grado de asociación significativo entre el estilo gerencial consultivo y participativo, con los factores motivadores e higiénicos de la satisfacción laboral en el personal administrativo de la Administración Central de la UNMSM.

\section{MARCO TEÓRICO}

George R. Terry ha definido el liderazgo como la "actividad de influenciar en la gente para que se empeñe voluntariamente en el logro de los objetivos del grupo". Por grupo debe entenderse un grupo pequeño, un sector de la organización, una organización, una nación, etc. (Lazzati,1991, pág. 67).

Según Lazzati (1991), de tal definición surgen dos campos fundamentales de liderazgo relacionados a la organización:

- El proceso intelectual de concebir los objetivos de la organización.

- El factor humano, esto es, influenciar a la gente para que voluntariamente se empeñe en el logro de los objetivos.

Para Lazzati (1991), si se aceptan estas dos condiciones, puede concluirse que liderazgo y motivación se encuentran interrelacionados, en donde la primera mira al líder y la segunda a sus seguidores; que liderar es provocar motivación; que cualquier análisis de liderazgo tiene su correlato en materia de motivación, y viceversa. 
Casales define el liderazgo de la siguiente forma: "Se trata de aquel miembro que ejerce mayor influencia sobre los restantes integrantes de su grupo de manera espontánea, siendo capaz de motivarlos y guiarlos hacia la obtención de un objetivo o meta, y que desempeña un rol central en el desarrollo de las tareas y el funcionamiento grupal al realizar contribuciones de significación" (Casales, 1995, pág 39).

Romero (1993; citado por Salom y Barreat, 1998) define el liderazgo motivacional como la influencia que unas personas ejercen sobre otras basada en características personales particulares.

Así mismo, Morales (1994; citado por Salom y Barret, 1998) define operacionalmente a los líderes motivacionales como aquellos que poseen predominantemente excelencia, fortaleza, esperanza activa y flexibilidad.

Tannenbaum, Weschler y Masarik definen el liderazgo como "la influencia personal ejercida en una situación y dirigida - mediante el proceso de la comunicación- a conseguir una o varias metas particulares". (Hersey, Blanchard y Johnson, 1997, pág. 91).

Igualmente, Koontz y O’Donnell afirman que "el liderazgo consiste en influir en la gente para que se una en la consecución de cierta meta en común”. (Hersey, Blanchard y Johnson, 1997, pág. 91).

Dentro de su extenso trabajo Likert (1961) ha identificado cuatro estilos gerenciales más comunes (en los últimos años hace referencia a un quinto estilo) que son los que se presentan a través de las organizaciones.

\section{TEORÍA DE RENSIS LIKERT}

Likert (1968) determinó la existencia de cuatro sistemas administrativos basándose en las características y estilos de la dirección presente en una organización: Estas cuatro son: autoritarismocoercitivo, autoritario-benevolente, participativo y consultivo. Estos encabezamientos o epígrafes fueron presentados por primera vez en su obra New Patterns of Management (1961). Posteriormente fueron cambiados en El factor humano en la empresa: Su dirección y valoración (1968), respectivamente, a sistema 1 , sistema 2 , sistema 3 y sistema 4; que se presenta a continuación:

Sistema 1: Autoritario-coercitivo: Bajo este sistema de liderazgo, la administración no confía en sus empleados y rara vez los hace participar en algún aspecto de la toma de decisiones. El grueso de las decisiones y el establecimiento de las metas de la organización se toman arriba y se despachan hacia abajo por la cadena de mando. Los empleados se ven obligados a trabajar con miedo, amenazas, castigos y ocasionales recompensas. La satisfacción de necesidades se encuentra en los niveles fisiológico y de seguridad. Los limitados intercambios entre la administración y los procesos de control están muy concentrados en la alta dirección, por lo común se desarrolla una organización informal en oposición a las metas de la empresa.

Sistema 2: Autoritario-benevolente: La administración obsequia una confianza condescendiente a sus empleados, como la del amo con sus servidores. El grueso de las decisiones y el establecimiento de las metas de la organización se hacen hacia arriba, pero en los niveles inferiores se toman muchas decisiones de acuerdo con un marco preestablecido. Se dan recompensas y algunos castigos reales o potenciales para motivar a los trabajadores. Cualquier intercambio tiene lugar con la indulgencia de la administración y el temor y la cautela de parte de los empleados. Aunque el proceso de control sigue concentrado en la alta gerencia, algo se delega a los niveles medios y bajos. Habitualmente aparece una organización informal, pero no siempre se opone a las metas formales de la empresa.

Sistema 3: Consultivo: La administración confía, pero no del todo, en sus empleados. Las políticas y las cesiones generales se mantienen arriba, pero se permite que los trabajadores tomen decisiones particulares en niveles inferiores. La comunicación fluye en ambos sentidos de la jerarquía. Para motivar a los trabajadores, se dan recompensas, castigos ocasionales y alguna participación. Hay un grado moderado de intercambios, a menudo con suficiente confianza. Se delegan aspectos significativos del proceso de control, con sentimientos de responsabilidad tanto en los niveles superiores como en los inferiores. Puede parecer una organización informal, que bien respalde o bien se resista en parte a las metas de las empresas.

Sistema 4: Participativo: La administración tiene una confianza plena en sus empleados. La toma de decisiones está repartida en toda la organización y, con todo, bien integrada. La comunicación no solo fluye en sentido vertical, 
sino también horizontal entre los compañeros. Los trabajadores están motivados por la participación y el compromiso en el desarrollo de premios económicos, el establecimiento de metas, la mejora de los métodos y la valoración de los progresos rumbo a las metas. Los intercambios entre la administración y los empleados son extensos y amistosos, con un alto grado de confianza. La responsabilidad del proceso de control está muy distribuida, e incluso las unidades inferiores participan por completo. Las organizaciones formal e informal suelen ser la misma, por lo que todas las fuerzas sociales respaldan los empeños por lograr las metas establecidas.

Las características generales de cada uno de estos estilos son:

\section{Sistema 1}

- Opera dentro del estilo.

- Se basa en el temor y las amenazas.

- La comunicación es desde arriba y hacia abajo.

- Existe un importante distanciamiento psicológico entre el superior y el subordinado.

- Las decisiones se toman en la cúspide de la organización.

\section{Sistema 2}

- Opera dentro del estilo autoritario benevolente.

- Consigue cumplimiento a través de las recompensas.

- Las actitudes del personal son de subordinación hacia sus superiores.

- La información fluye generalmente de arriba hacia abajo y muy ocasionalmente fluye hacia arriba, limitándose a aquellas cosas que el jefe quiere escuchar.

- Las decisiones vitales y de política son tomadas en la cúspide de la empresa, pero está prescripto (formalmente) lo (poco) que puede ser delegado a los subordinados.

\section{Sistema 3}

- Opera dentro del esquema consultivo.

- Consigue cumplimiento como consecuencia de las recompensas, ocasionalmente hace uso de ciertos castigos y se busca algún involucramiento.
- El flujo de la información comienza a transformarse en comunicación; es tanto hacia arriba como hacia abajo. La comunicación hacia arriba que no incluye lo que el jefe quiere escuchar es proporcionada en pequeñas cantidades y en forma muy cautelosa.

- Las decisiones vitales y de política son tomadas en la cúspide de la empresa y los subordinados pueden tener una cierta cantidad de influencia sobre lo que sucede dentro de sus unidades.

- Los subordinados pueden decidir sobre aspectos relacionados con su trabajo.

\section{Sistema 4}

- Opera bajo el sistema de gerenciamiento grupal.

- La gerencia se maneja con recompensas económicas.

- La gerencia pone en práctica un sistema de participación grupal.

- La gerencia promueve el involucramiento del personal en establecer altos objetivos de performance.

- La gerencia promueve el desarrollo de nuevas metodologías y procesos en el trabajo.

- La comunicación fluye hacia abajo, hacia arriba y hacia ambos lados y se realiza sobre base cierta.

- Los superiores y subordinados están muy próximos entre sí desde el punto de vista psicológico.

- La toma de decisiones dentro de la organización es realizada a través de procesos grupales.

- Existe una superposición entre los grupos donde el superior de una unidad es el subordinado de otra.

\section{CONCEPTO DE MOTIVACIÓN}

- Lazzati define la motivación de la gente como “ el proceso por el cuál una persona genera energía y dirección hacia cierto objetivo cuyo logro se supone habrá de satisfacer la necesidad" (Lazzati, 1991, pág. 68).

- A esta definición le agrega dos condiciones desde el punto de vista de la organización: a) Que la persona tenga cierto grado de satisfacción general con la organización, sin perjuicio 
de la necesidad puntual insatisfecha inherente a la motivación específica; y b) Que el objetivo de la persona que atañe a la motivación de que se trate sea convergente con los objetivos de la organización (Lazzati, 1991).

- En cuanto a la relación entre motivación y satisfacción, existe confusión en algunos administradores al pensar que son términos similares. Al respecto, Lawer III (1990) opina que no lo son: "De hecho, son muy diferentes. La motivación está influida por las consideraciones del futuro a propósito de la relación entre desempeño y recompensas, mientras que la satisfacción se refiere a los sentimientos de la gente hacia las recompensas que recibe. Así, la satisfacción es una consecuencia de los acontecimientos pasados, mientras que la motivación es el resultado de las expectativas del porvenir" (Lawer III, 1990; citado por Hersey, Blanchard y Johnson, 1997, pág. 84).

\section{TEORÍA DE LOS DOS FACTORES DE HERZBERG}

- Por lo tanto, los esfuerzos por mejorar el desempeño futuro, concentrándose en las recompensas y los beneficios del pasado, muestran una falta de entendimiento del carácter de la motivación (Hersey, Blanchard y Johnson; 1997).

- La inquietud de Herzberg (1976) por la variable "satisfacción" lo llevó a liderar un trabajo de investigación donde participaron unos 200 profesionales en su gran mayoría contadores e ingenieros en la ciudad de Pittsburg, a quienes se les solicitó relataran -dentro de una serie de sucesos- aquellas situaciones en que se sintieron muy bien como también en aquellas otras donde no estaban satisfechos. Se realizó una tipología de eventos que, posteriormente, pudieron relacionarse con "satisfacción en el trabajo" e "insatisfacción en el trabajo".

- Y de aquí surge una serie de componentes que no se condicen con lo que el estado del arte de las ciencias del comportamiento tenía registrado hasta ese entonces. Frederick Herzberg (1976) encontró algunas evidencias que resultaron ser un hallazgo muy importante, por las implicancias que tienen para las organizaciones en su marcha hacia la eficiencia, cambio y desarrollo organizacional.

- Encontró que cinco factores estaban fuertemente relacionados con la satisfacción en el trabajo, los que son detalladas a continuación:

\section{Logro.}

2. Reconocimiento.

3. Cuán atrayente era el trabajo en sí mismo.

4. Responsabilidad.

5. Potencial de crecimiento.

Por otro lado, encontró también evidencia de que la carencia de estos cinco factores no era frecuentemente mencionada como una fuente de insatisfacción. Más bien, lo que encontró Herzberg (1976) es que las razones de la insatisfacción en el trabajo estaban más bien relacionadas con otros factores distintos de los arriba mencionados:

a. La política general de la empresa y su "administración".

b. Supervisión.

c. Salarios.

d. Condiciones de trabajo.

e. Relaciones interpersonales.

Entre otras cosas esto significa que si estoy interesado en mejorar la productividad y la eficiencia por más que empuje "las fuerzas a favor del cambio" (Kurt Lewin) y entre ellas están las que se mencionan bajo a, b, c, d, y e, es muy improbable que aumente la satisfacción del empleado y que este, a su vez, aumente su fuerza motivadora. Es muy probable que se ha de encontrar mejores resultados teniendo en cuenta las variables mencionadas bajo 1 , $2,3,4$ y 5 .

Es aquí donde Frederick Herzberg (1976) también toma en cuenta las conclusiones del excelente trabajo realizado por los investigadores de Ohio State University, cuando dilucidan "lo que hace el líder eficiente". Usualmente los investigadores -y también los académicos y practitioners- dividían sus opiniones respecto de si el líder eficiente se orientaba hacia el trabajo o si lo hacía hacia la persona. Los investigadores de Ohio State University descubrieron que estas -orientación hacia las personas u orientación hacia el trabajo- no eran polos opuestos de una misma dimensión, sino más, bien eran dos dimensiones distintas. Y Herzberg (1976) penetra en esta ruta, al estudiar satisfacción en el trabajo, sugiriendo que también aquí esta variable tiene dos dimensiones distintas, y no polos opuestos de una sola. 


\section{MÉTODO DE INVESTIGACIÓN}

El método empleado en la presente investigación es el descriptivo bivariable. Es descriptivo porque pretende caracterizar la situación de las dos variables que aborda la investigación en la población y muestra estudiadas.

La variable independiente, estilos gerenciales; y la dependiente, el nivel de satisfacción laboral de los colaboradores.

Los estilos gerenciales autoritario-coercitivo y autoritario-benevolente se han agrupado para ser asociados ambos con la variable dependiente satisfacción laboral. Igualmente se han agrupado los estilos consultivo y participativo para su respectiva asociación con la variable dependiente satisfacción laboral.

\section{RESULTADOS DE INVESTIGACIÓN RESULTADOS GENERALES}

Relación entre la satisfacción laboral y los estilos gerenciales

en el personal administrativo de la Dirección General de Administración de la UNMSM.

\begin{tabular}{|c|c|c|c|c|}
\hline \multirow{2}{*}{$\begin{array}{c}\text { Estilos Geren- } \\
\text { ciales }\end{array}$} & \multicolumn{2}{|l|}{ Satisfacción Laboral } & \multicolumn{2}{|c|}{ Análisis } \\
\cline { 2 - 5 } & Motivador & Higiénico & $\begin{array}{c}\text { Valor } \\
x^{2}\end{array}$ & $\begin{array}{c}P \\
\text { valor }\end{array}$ \\
\hline $\begin{array}{c}\text { Consultivo y } \\
\text { Participativo }\end{array}$ & 111 & 35 & & \\
\hline $\begin{array}{c}\text { Autoritario y } \\
\text { Benevolente }\end{array}$ & 60 & 44 & 9.446 & 0.002 \\
& & & & \\
\hline
\end{tabular}

Como el valor de significancia $(p=0.002)$ es menor 0.05 , podemos concluir que existe relación entre los estilos gerenciales con la satisfacción laboral en el personal administrativo de la Dirección General de Administración de la UNMSM, con un nivel de confianza del 95 por ciento.

\section{Gráfico № 1}

Frecuencias de la satisfacción laboral según estilos gerenciales en el personal administrativo de la Dirección General de Administración de la UNMSM.

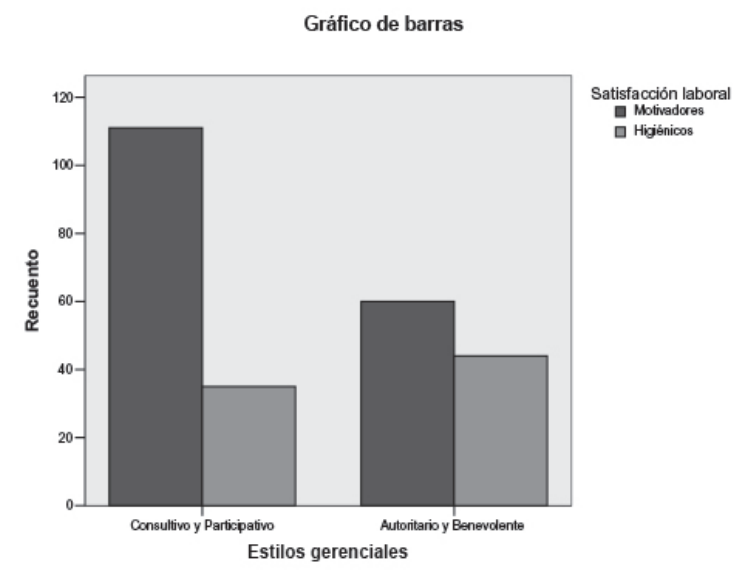

\section{DISCUSIÓN DE RESULTADOS \\ HIPÓTESIS GENERAL}

Con respecto a la hipótesis general que dice: existe relación significativa entre los estilos gerenciales y la satisfacción laboral en el personal administrativo de la Administración Central de la UNMSM.

Contrastación: Se acepta la hipótesis general al probarse que ambos estilos se relacionan con la satisfacción laboral.

Si relacionamos los estilos gerenciales con la satisfacción laboral según dependencia, los resultados son los siguientes: Existe relación entre los estilos gerenciales y la satisfacción laboral en las dependencias de Operaciones y Mantenimiento y Bienestar Universitario.

No existe relación entre los estilos gerenciales y la satisfacción laboral en las siguientes dependencias: Oficina General de Recursos Humanos, Oficina General de Economía y Oficina General de Infraestructura.

Interpretación: Siguiendo a Herzberg, cuando una persona está motivada por algo - por ejemplo, su trabajo le brinda la oportunidad de autorrealización personal - entonces su trabajo se convierte en una fuente de satisfacción. Por el contrario, si su trabajo no le brinda la oportunidad de logro, entonces la persona carecerá de motivación para trabajar y estará no-satisfecha, que es diferente de 
estar insatisfecha. Si esta persona encuentra en su trabajo un entorno adverso -por ejemplo, condiciones físicas desagradable- entonces su trabajo pasará a ser una fuente de insatisfacción, porque los factores no están adecuadamente resueltos.

En la relación entre estilos gerenciales y satisfacción laboral, hemos encontrado que el estilo consultivo y participativo es el que reporta mejores resultados de satisfacción laboral, superiores al estilo autoritario coercitivo y autoritario benevolente, explicable en gran parte porque el primero de ellos promueve factores de motivación intrínseca con indicadores más altos, como el reconocimiento seguido por el trabajo en sí mismo y por el crecimiento que equivale a decir la posibilidad de obtener logros y que estos sean reconocidos. Estos indicadores son los que realmente proporcionan motivación en el trabajador.

En cambio, el estilo gerencial autoritario coercitivo y autoritario benevolente reporta menores resultados de satisfacción al concentrar más la toma de decisiones, disminuir la comunicación descendente y ejercer menor confianza en los empleados, brindándoles poca o casi nula participación en algún aspecto de la toma de decisiones; y poco reconocimiento origina menor satisfacción laboral tanto a nivel de factores motivadores como de factores higiénicos inclusive.

\section{HIPÓTESIS ESPECIÍFICAS}

\section{Hipótesis específica 1}

Con respecto a la hipótesis específica 1 que dice: Existe un grado de asociación no significativo entre el estilo gerencial autoritario explotador y autoritario benevolente con los factores motivadores e higiénicos de la satisfacción laboral en el personal administrativo de la Administración Central de la UNMSM.

Contrastación: Se acepta la hipótesis específica 1 , al comprobarse que existe un grado de asociación irrelevante o poco significativo entre el estilo gerencial autoritario explotador y autoritario benevolente con los factores motivadores e higiénicos de la satisfacción laboral en el personal administrativo de la Administración Central de la UNMSM.

Interpretación: Esto podría explicarse según Likert (1961) porque el tipo de liderazgo y supervisión que se basa en poner permanente presión sobre los subordinados, quienes a su vez están comprometidos con cada vez más y más subtareas especializadas, lo que refuerza el gerenciamiento en función de "tener permanentemente ocupadas a las personas", no es el más efectivo y eficiente. Este tipo de supervisión del líder, al que Likert denomina supervisión "centrada en la tarea”, puede producir resultados medianamente satisfactorios solamente en el corto plazo y sus consecuencias disfuncionales dentro de su propio grupo de trabajo, como así también respecto de otros y la organización como un conjunto, se han de mostrar muy rápidamente. Los subordinados, a su vez, con ese estilo de liderazgo, tendrán menos posibilidades de desarrollar iniciativas. En los indicadores de satisfacción hallados en este estilo, encontramos que son sustantivamente menores que los del estilo consultivo participativo, tanto a nivel de factores motivadores tales como logro, reconocimiento, trabajo en sí mismo, reconocimiento, responsabilidad, ascenso y crecimiento, como en los factores higiénicos que evitan la insatisfacción con indicadores tales como sueldo, estatus, vida personal, seguridad, relaciones con el jefe y compañeros.

\section{Hipótesis específica 2}

Con respecto a la hipótesis específica 1 que dice:

Existe un grado de asociación significativo entre el estilo gerencial consultivo participativo, con los factores motivadores e higiénicos de la satisfacción laboral en el personal administrativo de la Administración Central de la UNMSM.

Contrastación: Se acepta la hipótesis específica 2 al comprobarse que existe un grado de asociación, aunque bajo, entre el estilo gerencial consultivo y participativo con los factores motivadores e higiénicos de la satisfacción laboral en el personal administrativo de la Administración Central de la UNMSM.

Interpretación: Estos resultados se pueden explicar desde la teoría Likert, que señala que, estilo consultivo aumenta el grado de satisfacción de los colaboradores, ya que permite que los trabajadores tomen decisiones particulares en niveles inferiores y que la comunicación fluya en ambos sentidos de la jerarquía. Motiva a los trabajadores, que reciben recompensas y participación. Hay un grado moderado de intercambios, a menudo con suficiente confianza. Se delegan aspectos significativos del proceso de control, 
con sentimientos de responsabilidad tanto en los niveles superiores como en los inferiores. Puede parecer una organización informal, que bien respalde o bien se resista en parte a las metas de las empresas. Este estilo de liderazgo está originando entonces mejores resultados de satisfacción laboral, aunque no en un grado significativamente mayor de satisfacción laboral.

Esto se cumpliría tanto a nivel de los indicadores de los factores motivacionales como de los higiénicos, esto quiere decir que el estilo desarrolla un mejor manejo de la motivación tanto intrínseca, la que verdaderamente origina satisfacción, como en la extrínseca, la que evita que exista insatisfacción.

\section{CONCLUSIONES}

1. Se acepta la hipótesis general al probarse que existe relación significativa entre los estilos gerenciales y la satisfacción laboral en el personal administrativo de la Administración Central de la UNMSM. Ambos estilos se relacionan con la satisfacción laboral.

Los estilos consultivo y participativo mantienen una relación positiva con la satisfacción laboral, tanto en los factores e indicadores motivadores como en los higiénicos.

Los estilos autoritario coercitivo y autoritario benevolente mantienen relaciones más débiles con la satisfacción laboral, tanto en los factores e indicadores motivadores como en los higiénicos.

2. Se acepta la hipótesis específica 1 al probarse que existe un grado de asociación irrelevante o poco significativo entre los estilos gerenciales autoritario -coercitivo y autoritario- benevolente y la satisfacción laboral en el personal administrativo de la Administración Central de la UNMSM. Esto significa que el estilo gerencial autoritario-coercitivo y autoritariobenevolente se asocia en forma débil con la satisfacción laboral en el personal administrativo de la Administración Central de la UNMSM. Los indicadores de satisfacción hallados en este estilo son sustantivamente menores que los del estilo consultivo participativo tanto a nivel de factores motivadores como a nivel de factores higiénicos.

3. Se acepta la hipótesis específica 2 al probarse existe un grado de asociación significativo, aunque bajo, entre los estilos gerenciales consultivo y participativo y la satisfacción laboral en el personal de la Administración Central de la UNMSM. Esto significa que el estilo gerencial consultivo y participativo se asocia mejor con la satisfacción laboral, aunque en un grado no muy alto, en el personal administrativo de la Administración Central de la UNMSM. Este estilo de liderazgo está originando mejores resultados de satisfacción laboral, tanto a nivel de los indicadores de los factores motivacionales como de los higiénicos. Esto quiere decir que este estilo desarrolla un mejor manejo de la motivación extrínseca como intrínseca, la que verdaderamente origina satisfacción, como en la extrínseca la que evita que exista insatisfacción.

\section{REFERENCIAS BIBLIOGRÁFICAS}

Álvarez De Mon, S. (1997). Liderazgo transformador. Harvard Deusto Business Review, 79, 49-57.

Arana, W (2005). Diseño y validación de un modelo para la identificación y medición de los factores motivacionales delos trabajadores según la teoría de Fredeick Herzberg. FCA UNMSM

Bennis, W. (1984). The competencies of leadership. Training and development journal. En: Robbins, S. Comportamiento organizacional, México, Prentice - Hall Hispanoamericana, 380-419.

Brooke, P; Russell, D. y Price, J. (1988). Discriminant validation of measures of job satisfaction, job involvement, and organizational commitment. Journal of appliedpsychology. En: Pérez, I. Satisfacción laboral y comportamientos organizacionales. Revistainteramericana de psicologíaocupacional, 14 , (1), 7-23.

Bryson, J. (1981). A perspective on planning and crisis in the public sector. Strategic management journal. En: Gibson, D. Las organizaciones, Lugar, Addison Wesley Interamericana, 388-411.

Butterfield, E (2001). Las Emociones y Lo Social en las Empresas. Congreso organizado por TheOrganizationDevelopmentInstitute International, Latinoamérica. En: www. gestiopolis.com 
Casales, J. (1995). Estilos de dirección, liderazgo y productividad grupal. Revista interamericana de psicología ocupacional,14, (1), 25-53.

Conger, J. (1989). Thecharismatic leader. En: Gibson, D. Las organizaciones, Lugar, Addison Wesley Interamericana, 388-411.

Conger, J. y Kanungo, R. (1988). Charismaticleadership. En: Robbins, S. Comportamiento organizacional, México, Prentice - Hall Hispanoamericana, 405-410.

Covey, Stephen (1990). Liderazgo Centrado en Principios” Nueva York, Simon\&Schuster

Fernández, J. (1999). Un modelo para la dirección de grupos de trabajo. Liderazgo natural. Cuadernos de management. Para una dirección eficaz,86 (438), 67-82.

Flores, J. (1996). El comportamiento humano en las organizaciones. Lima. Universidad del Pacífico.

García, M. y De María, F. (1981). Administración y desarrollo gerencial. México, D.F.: Diana. 3era. Ed.

Gibson, D. (1996). Las organizaciones. AddisonWesley Iberoamericana.

Hackman, J; Richard y otros. (1975). A new strategy for job enrichment. California managementreview. En: (b), 348-359.

Herman, R. (1997). Turbulencia. Retos y oportunidades en el mundo del trabajo. ¿Está usted preparado para el futuro? Santafé de Bogotá: McGraw-Hill Interamericana.

Hersey, P; Blanchard, K. y Johnson, D. ( ). Administración del comportamiento organizacional. Liderazgo situacional. México, D.F.: Prentice-Hall Hispanoamericana. 7ma. Ed.

Herzberg, F. (1968). One more time: How do you motivate employees? Harvard businessreview. En: Pérez, I. La satisfacción en el trabajo: Un enfoque para su estudio. Revista Interamericana de Psicología Ocupacional, 15, (2), 65-79.

House, R. (1976). Theory of charismaticleadership. En: Gibson, D. Las organizaciones, Lugar, Addison Wesley Interamericana, 388-411.

Lawer III, E. (1990). High involvement management. En: Hersey, P; Blanchard, K. y Johnson, D. Administración del comportamiento organizacional. Liderazgo situacional, México, Prentice-Hall Hispanoamericana, 84-85.

Lazzati, S. (1991). Management. Funciones, estilos y desarrollo. Buenos Aires: MACCHI.

Lewin, K; Lippitt, R. y White, R. (1939). Patterns of aggressive behavior in experimentally created social climates. Journal of social psychology. En (6), 175-184.

Likert, R. (1968). El factor humano en la empresa: Su dirección y valoración. Bilbao: Deusto. 1 ra. Ed.

Likert, Rensis (1961). Un Nuevo Método de Gestión y Dirección. Bilbao. Deusto

Locke, E. (1969). What is job satisfaction. Organizational behavior and human performance. En: Pérez, I. La satisfacción en el trabajo: Un enfoque para su estudio. Revista interamericana de psicología ocupacional,15, (2), 65-79.

López Más Julio.(2005) Motivación laboral y Gestión de recursos humanos en la teoría de Frederick Herzberg ${ }^{\prime}$ 'Rev. Tercer Milenio N 15 F.C.A. U.N.M.SM.

Lord, R; DeVader, C. y Alliger, G. (1986). A metaanalysis of the relation between personality traits and leadership perceptions: An application of validity generalization procedures. Journal of applied psychology. En: Robbins, S. Comportamientoorganizacional, México, Prentice - Hall Hispanoamericana, 380-419.

MacFarlin, D. Y Rice, R. (1992). The role of facet importance as a moderator in job satisfaction process. Journal of organizationalbehavior. En: Pérez, I. (1996). La satisfacción en el trabajo: Un enfoque para su estudio. Revista Interamericana de Psicología Ocupacional, $15,(2), 65-79$.

Meindl, J; Elvilich, S. Y Dukerich, J. (1985). The romance of leadership. Administrative science quarterly. En: Robbins, S. Comportamiento organizacional, México, Prentice - Hall Hispanoamericana, 380-419.

Murdick, R; y Munson, J. (1988). Sistemas de información administrativa. México, D.F.: Prentice Hall Hispanoamericana.2da. Ed.

Pérez, I. (1995). Satisfacción laboral y comportamientos organizacionales. Revista Interamericana de Psicología Ocupacional,14, (1), 7-23. 
Pérez, I. (1996). La satisfacción en el trabajo: Un enfoque para su estudio. Revista interamericana de psicología ocupacional,15, (2), 65-79.

Podsakoff, P; Tudor, W. Y Skov, R. (1982). Effect of leader contingent and non - contingent reward and punishment behavior on subordinate performance and satisfaction. Academy of management journal. En: Gibson, D. Las organizaciones, Lugar, Addison Wesley Interamericana, 407-411.

Powell, G. y Butterfield, D. (1984). The high-high leader again. Group and organizationstudies. En: Robbins, S. Comportamiento organizacional, México, Prentice - Hall Hispanoamericana, 380-419.

Robbins, S. (1993). Comportamiento organizacional. México, D.F.: Prentice - Hall Hispanoamericana.5ta. Ed.

Robbins, S. y Coulter, M. (1996). Administración. México, D.F.: Prentice Hall Hispanoamericana. 5ta. Ed.

Robbins, S. y De Cenzo, D. (1996). Fundamentos de administración. Conceptos esenciales y aplicaciones. México, D.F.: Prentice Hall Hispanoamericana. 1era. Ed.

Rusbult, C. y Lowery, D. (1985). When bureauerats get the blues. Journal of Applied Social
Psychology. En: Robbins, S. Comportamiento organizacional, México, Prentice - Hall Hispanoamericana, 187-197.

Salom, C. y Barret, Y. (1998). Estrategias de influencia usadas por los líderes motivacionales. Revista interamericana de psicología ocupacional, 17 (2), 5-13.

Sharma,S. (1995). Motivation.University of Toronto: Faculty of InformationStudies. Disponible en: http://choo./LIS.utoronto. ca/FIS/Courses/LIS1230/LIS1230sharma/ motive/.html

Stogdill, R. (1948). Personal factors associated with leadership: Survey of the literature. Journal of applied psychology. En: (6), 176 -177 .

Vroom, V. y Yetton P. (1973). Leadership and decision making. En: Gibson, D. Las organizaciones, México, Addison Wesley Interamericana, 391-397.

Weber, M. (1947). The theory of social and economic organization. En: Gibson, D. Las organizaciones, México, Addison Wesley Interamericana, 388-411.

Williams, R. y Blackler, F. (1971). Motives and behavior at work. London: Cox \&Wyman. 1era Ed. Estatuto de la UNMSM 
